\title{
Newborn screening for severe combined immunodeficiency: a primer for clinicians
}

\author{
Catherine M. Biggs MD, Elie Haddad MD PhD, Thomas B. Issekutz MD, Chaim M. Roifman MD, \\ Stuart E. Turvey MBBS DPhil
}

Cite as: CMAJ 2017 December 18;189:E1551-7. doi: 10.1503/cmaj.170561

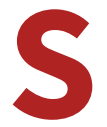

evere combined immunodeficiency is caused by genetic defects that profoundly impair development of the immune system. ${ }^{1,2}$ The condition is fatal early in life if affected infants do not receive therapy to restore immune function in the form of hematopoietic stem cell transplantation, enzyme replacement therapy or gene therapy. Infants with severe combined immunodeficiency commonly appear healthy at birth; consequently, the condition often presents only when the child has already had many infections and secondary organ damage. ${ }^{3,4}$ Early diagnosis is essential, as patients who receive a transplant before 3.5 months of age have the best outcomes.,

In Canada, severe combined immunodeficiency is diagnosed at a mean age of 4.2 months and carries a mortality rate of $30 \%$, with nearly $60 \%$ of deaths caused by infection before patients are able to receive a transplant. ${ }^{7}$ The clear need for early diagnosis and treatment led to the development of a newborn screen for severe combined immunodeficiency, known as the T-cell receptor excision circle assay. ${ }^{8}$ This assay was established in 2005 and is carried out on dried blood spots (also known as the Guthrie card), which are already taken as part of the routine newborn screen. ${ }^{9}$ In 2013, a newborn screening program for severe combined immunodeficiency was introduced in Ontario, following an application made by Immunodeficiency Canada, a national nonprofit advocacy organization with a mission to study and cure inherited immunodeficiency. The screening program has since expanded to the Maritime provinces and is being established in several other provinces and territories.

\section{Box 1: Evidence used in this review}

To augment our clinical expertise, we searched PubMed and Web of Science for "newborn screening for severe combined immunodeficiency," and various combinations of "newborn screening," "neonatal screening," "severe combined immunodeficiency," "SCID," and "TREC." We also studied seminal papers on the diagnosis and treatment of primary immunodeficiencies. Recent articles and those with the most robust level of evidence, such as large-scale population-based studies and those using Markov and decision analysis models to estimate cost-effectiveness of newborn screening for severe combined immunodeficiency, were selected. Of the 126 papers reviewed, 42 were used in this review.

\section{KEY POINTS}

- Severe combined immunodeficiency is a medical emergency that can potentially be cured by hematopoietic stem cell transplantation or gene therapy (in specific diseases).

- Outcomes for severe combined immunodeficiency are greatly improved by early diagnosis and treatment.

- The newborn screen for severe combined immunodeficiency evaluates for T-cell lymphopenia using a T-cell receptor excision circle assay, which can be performed on the dried newborn blood spot.

- Infants with a positive newborn screen for severe combined immunodeficiency require referral to a clinical immunologist, as well as precautionary measures against infection while they undergo evaluation.

This review informs physicians who may treat newborns in their practice (e.g., family physicians, obstetricians, pediatricians) on how to approach patients and counsel families who are faced with an abnormal screen for severe combined immunodeficiency. We describe the epidemiology, diagnosis and screening of this disorder and provide a brief summary of available treatments (Box 1).

\section{What is severe combined immunodeficiency?}

The T lymphocyte, or T cell, is an essential part of the immune response: it protects against infectious pathogens, contributes to immunity against cancer and aids in preventing self-reactive processes such as autoimmunity. Because the T cell activates other cells of the immune system (B cells, monocytes), a defect in $T$ cells confers an associated defect in B cells - hence the term "combined" immune deficiency. Severe combined immunodeficiency is caused by a variety of genetic defects that severely impair the development and function of T cells. ${ }^{2}$ The incidence of severe combined immunodeficiency was previously thought to be 1:100000. Improved recognition through newborn screening has enabled a more accurate estimate of 1:58000. ${ }^{10,11} \mathrm{~A}$ Canadian national surveillance study found a high incidence of the disorder in First Nations, Métis and Inuit Canadian children of 1:23000 
live births, and an incidence of 1:71000 live births in non-First Nations, Métis and Inuit Canadian children. ${ }^{7}$ Unless they have a genetic syndrome that causes other congenital anomalies, infants with severe combined immunodeficiency appear healthy at birth, which contributes to the challenge of early recognition. In the absence of a family history of the disorder or detection through a newborn screening program, the average age of clinical presentation is six months. ${ }^{12}$

\section{How is severe combined immunodeficiency diagnosed?}

Although physicians may find newborns with severe combined immunodeficiency to have no unusual findings at all, some typical presenting features include recurrent, severe or opportunistic infections, failure to thrive, absent tonsils and lack of peripheral lymph nodes. ${ }^{12,13}$ Affected infants may experience viral, fungal or bacterial infections, most commonly oral candidiasis, viral illnesses (particularly severe cytomegalovirus infection) or Pneumocystis jiroveci pneumonia. ${ }^{12}$ In addition to infections, infants with severe combined immunodeficiency can develop graft-versus-host disease, a life-threatening complication caused by foreign leukocytes attacking the infants' tissues. Graft-versus-host disease can develop if these infants are exposed to nonirradiated blood products, or from the presence of maternal T cells that have crossed the placenta. ${ }^{2,9}$ Infants with variant forms of severe combined

Box 2: Management of severe combined immunodeficiency

\section{Infection prevention}

- Infection control measures ${ }^{16}$

- Immunoglobulin replacement therapy

- Administration of palivizumab during respiratory syncytial virus season $^{17}$

- Trimethoprim-sulfamethoxazole prophylaxis starting at 6 weeks of age

Monitoring for complications

- Screening for infectious, autoimmune or other complications that may be related to patient's underlying genetic defect

- Comprehensive evaluation of signs or symptoms concerning for infection or autoimmunity, followed by prompt beginning of treatment

Curative therapy

- Hematopoietic stem cell transplantation: replacement of the affected patient's blood cell lines with those of a healthy donor ${ }^{17,18}$

- Enzyme replacement therapy: a treatment that replaces the defective enzyme causing SCID; may be considered in some specific genetic causes of $\mathrm{SCID}^{17,18}$

- Gene therapy: a treatment that corrects the defective gene; may be considered in some specific genetic causes of SCID ${ }^{17,18}$

Psychosocial and nutritional support

- Social work involvement to connect families with services

- Provide families with resources related to primary immunodeficiency (Box 3)

- Nutritional support to ensure appropriate growth and nutrition

Note: SCID = severe combined immunodeficiency. immunodeficiency, such as Omenn syndrome, can have features of immune dysregulation, which may include eosinophilia, skin rash, organomegaly, diarrhea and metabolic derangements. ${ }^{13,14}$

Investigations may show a low or normal absolute lymphocyte count and, in some cases, an absent thymic shadow on chest $\mathrm{x}$-ray. ${ }^{12} \mathrm{~A}$ low absolute lymphocyte count may be detected on a simple complete blood count and differential, and lymphopenia in infancy should not be ignored. The diagnosis of severe combined immunodeficiency is confirmed by the findings of either of the following: very low or absent T-cell counts and profoundly impaired $\mathrm{T}$-cell function, as measured by assessing the proliferation of $\mathrm{T}$ cells in response to stimuli; or maternal engraftment, defined by the presence of maternal T cells in the infant's circulation. ${ }^{15}$

Although it is not required to make the diagnosis of severe combined immunodeficiency, identifying the underlying genetic cause is valuable. It aids in genetic counselling of family members and may influence the selection of therapy. A brief summary of the treatment of severe combined immunodeficiency is provided in Box 2. ${ }^{16-18}$

\section{Why is early diagnosis and treatment critical?}

The cost of delayed diagnosis of severe combined immunodeficiency is substantial, as survival outcomes vary greatly depending on the timing of treatment and infection status. Retrospective studies show that infants who receive a hematopoietic stem cell transplant within the first 3.5 months of life have a greater than $90 \%$ likelihood of survival, even in the absence of a matched sibling donor. ${ }^{5,6}$ For those who receive a transplant after 3.5 months of age, overall survival rate falls to $70 \%,{ }^{6}$ with rates varying between $50 \%$ and $90 \%$ depending on the infection status at the time of transplant ${ }^{5}$ and on the donor type. Because infants may appear outwardly healthy until they begin experiencing recurrent infections or failure to thrive, by the time the diagnosis is made, the early window of opportunity to achieve an optimal outcome has typically passed. Children may die from severe infection before definitive treatment, or face worse transplantation outcomes than those in whom the disease is identified earlier in life. ${ }^{4}$ This knowledge of the difference that early diagnosis makes regarding outcomes fuelled the search for a biomarker that could be used to screen for severe combined immunodeficiency in the newborn period, which led to the discovery of the T-cell receptor excision circle assay.

\section{What is the newborn screen and how well does it perform?}

The newborn screen for severe combined immunodeficiency uses quantitative polymerase chain reaction to measure the number of T-cell receptor excision circles that are present in the blood. ${ }^{9}$ These are circular DNA fragments that are created as a by-product of normal T-cell development in the thymus (Figure 1). ${ }^{19}$ The assay can be carried out on DNA eluted from the dried blood spots used for the newborn screen and is typically done in conjunction with quantitative polymerase chain reaction of a control gene to rule out technical issues as a cause for a low 
number of T-cell receptor excision circles. ${ }^{19}$ The number of T-cell receptor excision circles present is proportional to the number of unique $T$ cells being generated and can thus act as a biomarker for T-cell development. The screen detects conditions that lead to decreased thymic T-cell production in neonates and is therefore a screen for T-cell lymphopenia. Dried blood spots from infants who initially appeared healthy at birth and were later found to have severe combined immunodeficiency have shown few T-cell receptor excision circles. ${ }^{9}$ The screen was first implemented by the state of Wisconsin in 2008 and is now being done in most states within the United States, as well as in numerous other countries. ${ }^{10,20-24}$ Ontario was the first province in Canada to introduce the screen, and the program has been expanding to other regions of Canada. ${ }^{25}$ The cost-effectiveness of newborn screening for severe combined immunodeficiency is well established. ${ }^{26,27}$

The severe combined immunodeficiency newborn screen shows excellent diagnostic performance, with high sensitivity and specificity. ${ }^{20,28,29}$ Numerous case series demonstrate $100 \%$ sensitivity for identifying typical severe combined immunodeficiency - defined as those infants with a T-cell count lower than 300 cells $/ \mu \mathrm{L}$, or those with maternal engraftment. ${ }^{29}$ Specificity is also high, identified as $99.98 \%$ in a cohort of infants undergoing newborn screening for severe combined immunodeficiency. ${ }^{20} \mathrm{~A}$ systematic review of cohort studies on severe combined immunodeficiency newborn screening found a positive predictive value ranging from $0.8 \%$ to $11.2 \%$ for diagnosing typical severe combined immunodeficiency, and from $18.3 \%$ to $81 \%$ for identifying both typical severe combined immunodeficiency and infants with T-cell counts that are substantially low, but do not meet the severity threshold required for diagnosing severe combined immunodeficiency. ${ }^{29}$

The false-positive rate for the T-cell receptor excision circle assay refers to the percentage of infants with low $\mathrm{T}$-cell receptor excision circle results requiring follow-up T-cell enumeration, who ultimately have a normal T-cell number. ${ }^{10}$ This rate varies depending on the cut-off values used for what are considered both normal T-cell receptor excision circle values and normal T-cell numbers. Infants have abundant T cells (ranging from 2500 to $5500 / \mu \mathrm{L}$ ); different locations that do the newborn screen have used varying T-cell value cut-offs (typically 1500 or $2500 / \mu \mathrm{L}$ ), in combination with T-cell phenotypic analysis, to decide which infants require further evaluation. ${ }^{10,30}$ This has led to great variation in false-positive rates between centres. ${ }^{10}$ Although low false-
Healthy-appearing child

A



Healthy-appearing child with SCID

B

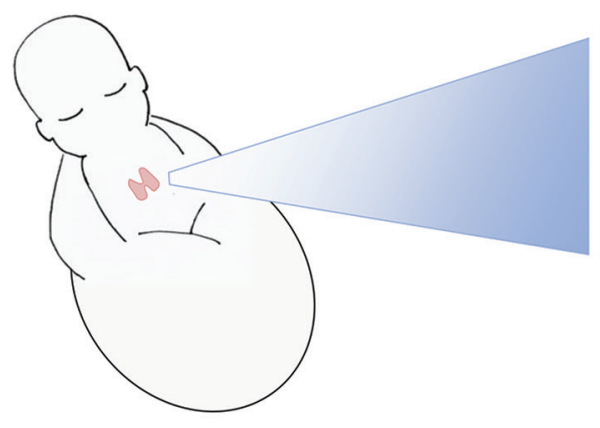

Normal T-cell production
Normal number of TRECS

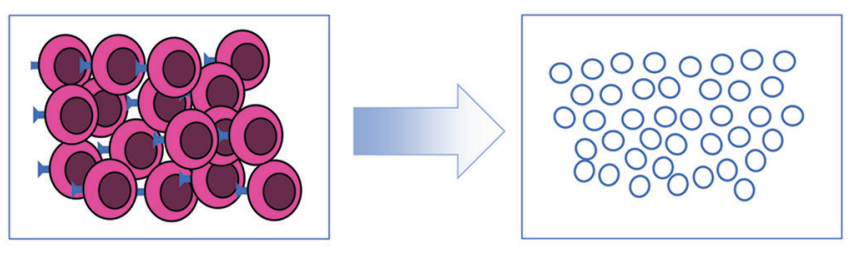

Very low or absent T-cell production



Figure 1: Pictorial explanation of T-cell receptor excision circle (TREC)-based newborn screen for severe combined immunodeficiency (SCID). The healthy infant (A) has an intact immune system; the thymus produces a normal repertoire of $T$ cells. As unique T cells are produced, TRECs are released into the systemic circulation and can be quantified on the newborn blood spot. Despite appearing healthy externally, the infant with SCID (B) has very low or absent T-cell production. This leads to undetectable numbers of TRECs on the newborn screen, facilitating early diagnosis of SCID. 
positive rates risk missing cases of severe combined immunodeficiency, higher rates may also lead to evaluating children who ultimately do not have the disorder. This is a challenge at present as Canada implements the newborn screen, and highlights the need for further research to determine the optimal cut-off values for T-cell receptor excision circles and T cells.

\section{Which other conditions may lead to an abnormal screen?}

Low or absent T-cell counts are the hallmark of severe combined immunodeficiency; however, T-cell lymphopenia can also be seen in other conditions (Table 1). Any condition or state that leads to decreased T-cell receptor production can result in an abnormal screen in newborns. Aside from severe combined immunodeficiency, other causes of T-cell lymphopenia include syndromic disorders and cytogenetic abnormalities, extreme prematurity and secondary $\mathrm{T}$-cell lymphopenia, which may be caused by congenital anomalies, T-cell losses from third spacing (that is, loss of lymphocytes into the interstitial space caused by vascular leakage or hydrops), neonatal leukemia, prenatal exposure to purine antagonists, and cardiac or gastrointestinal defects. ${ }^{10,23,31-33}$ Moreover, certain primary immunodeficiencies associated with T-cell dysfunction can also result in an abnormal T-cell receptor excision circle assay, despite the degree of T-cell lymphopenia not meeting the classic criteria for severe combined immunodeficiency. ${ }^{34-36}$

Table 1: Non-SCID conditions that may lead to an abnormal T-cell receptor excision circle-based newborn screen for SCID $^{10,23,31-36}$

\section{Non-SCID causes of T-cell}

lymphopenia

Examples

Syndromic disorders and congenital abnormalities

\begin{tabular}{|ll}
\hline & Trisomy 21 \\
\hline & DiGeorge syndrome \\
& CHARGE syndrome \\
\hline Ataxia-Telangiectasia syndrome \\
\hline Secondary T-cell lymphopenia & Cardiac or gastrointestinal anomalies \\
& Multiple congenital anomalies \\
\hline Neonatal leukemia \\
\hline T-cells losses into third space \\
(e.g., hydrops or vascular leakage) \\
$\begin{array}{l}\text { Prenatal exposure to purine } \\
\text { antagonists }\end{array}$
\end{tabular}

Preterm birth

Idiopathic T-cell lymphopenia

Combined immunodeficiencies with dysfunctional T cells

\section{DOCK8 deficiency}

Wiskott-Aldrich Syndrome

\section{Which forms of immunodeficiency are not identified by the newborn screen?}

Deleterious mutations in at least 20 genes have been associated with the development of severe combined immunodeficiency. ${ }^{37}$ Most of these are identifiable by the T-cell receptor excision circle assay; however, certain forms of the disorder can evade detection by the newborn screen. Examples include severe immunodeficiencies caused by ZAP70 and MHC class II deficiencies. These conditions are caused by defects in T-cell development that occur after the formation of the T-cell receptor and can therefore lead to variable results in T-cell receptor excision circle assays, ranging from low to normal..$^{38-41}$ Note that the assay does not screen for all primary immunodeficiencies. It will not identify immunodeficiencies in which $T$ cells are mildly affected or unaffected. ${ }^{37}$ These would include disorders of the innate immune system, such as complement deficiency or innate immune signalling defects, as well as primary B-cell disorders such as X-linked agammaglobulinemia. Although newborn screening for severe combined immunodeficiency is a huge advance for this extremely vulnerable patient population, it cannot replace "old-fashioned" clinical vigilance and acumen.

\section{What is the approach to a patient with an abnormal screen?}

Children with severe combined immunodeficiency are at risk for life-threatening infections; therefore, an abnormal newborn screen warrants prompt and careful evaluation (Figure 2). ${ }^{17}$ Infants should be referred to a pediatric immunologist for further evaluation as soon as an abnormal newborn screen has been identified and confirmed. Because the screen is not a definitive test, parents should receive reassurance that an abnormal result does not necessarily mean that their child has severe combined immunodeficiency. However, an abnormal screen should prompt further testing, comprising a complete blood count with differential, lymphocyte enumeration and analysis of T-cell phenotype and function. If this evaluation is consistent with classic severe combined immunodeficiency, immunologists may consider admission to hospital for protective isolation and full clinical evaluation. ${ }^{17}$ Otherwise, infants may remain at home while being evaluated, so long as they are well, without infectious symptoms; their home environment is safe; and parents are comfortable having a potentially ill infant at home. ${ }^{16}$

Those treating or caring for infants with suspected severe combined immunodeficiency should take the following precautions. Preventing community-acquired infections involves strict handwashing and avoiding public places or any sick contacts. The infant should not be exposed to other young children who may inadvertently transmit infections. Sterilizing bottles used to feed the infant and boiling ingestible water are important measures for preventing transmission of pathogens such as cryptosporidium. ${ }^{16}$ Infants or household contacts should not receive any live or liveattenuated vaccinations, including rotavirus; measles, mumps and rubella; varicella; and live-attenuated influenza. All blood products must be donor irradiated and leukocyte reduced. ${ }^{17}$ 
Cytomegalovirus infection has been associated with worse transplant outcomes if acquired by patients with severe combined immunodeficiency: ${ }^{42}$ if cytomegalovirus testing of blood products is possible, ensure that products the infant receives are cytomegalovirus negative. Cytomegalovirus is transmitted through body fluids, including oral secretions and breast milk, even when maternal carriers of cytomegalovirus are asymptomatic. Once a low level of T-cell receptor excision circles is confirmed, breastfeeding should be suspended until pediatric immunology evaluation has been undertaken; in the meantime, mothers who still wish to breastfeed may pump and store their breast milk until maternal cytomegalovirus status has been evaluated. Maternal cytomegalovirus status can be assessed by serology and, if negative, breastfeeding may resume. ${ }^{17,18}$

In cases of confirmed severe combined immunodeficiency, patients should be started on a prophylactic regimen to prevent infections, which typically includes trimethoprimsulfamethoxazole beginning at six weeks of age to prevent Pneumocystis jiroveci pneumonia, as well as immunoglobulin replacement therapy through administration of intravenous or subcutaneous immunoglobulins.

\section{What support should parents receive?}

Learning that their seemingly healthy newborn may have a lifethreatening diagnosis is devastating for new parents. These results arrive in the newborn period, when parents may be feeling particularly vulnerable, and when there is risk for developing postpartum depression. ${ }^{17}$ Sensitivity on the part of care providers is essential to help families cope while their child is undergoing evaluation. Practitioners should explain that the abnormal newborn screen alerts to the possibility of an immune system problem, but is not a definitive diagnosis. Emphasizing the screening nature of the test helps parents understand that although further workup is essential, false positives occur, and their child could still be identified as healthy. For those patients who are ultimately found to have severe combined immunodeficiency, therapies are available to treat the immunodeficiency, and those treated early in life have better outcomes.

Prompt referral to a pediatric immunologist is important, not only for an urgent immune evaluation, but also so that parents may receive further information on the screen and guidance on infection prevention. Parents may feel a range of emotions upon

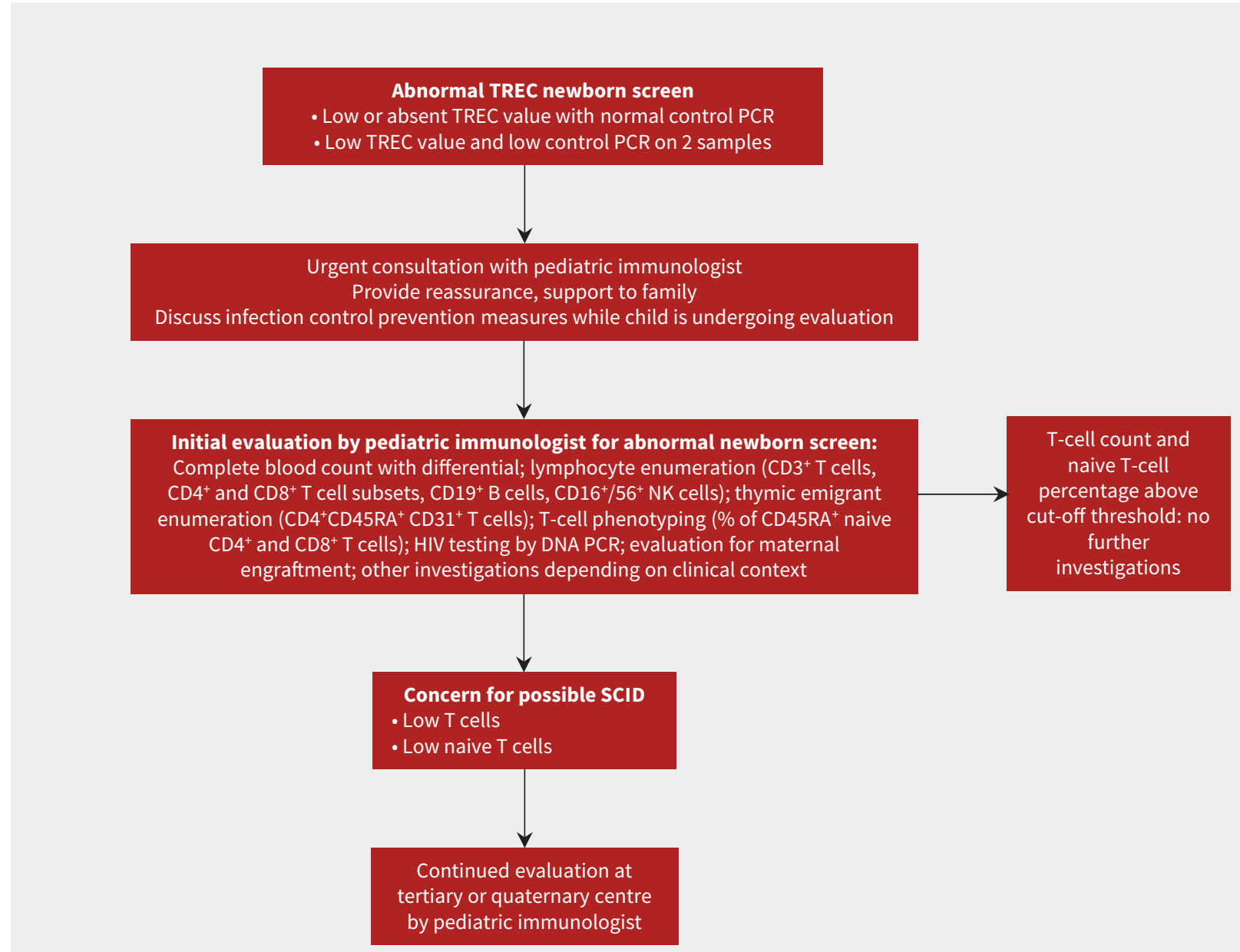

Figure 2: Suggested initial approach to a patient with an abnormal newborn screen for severe combined immunodeficiency $(\mathrm{SCID}) .{ }^{17} \mathrm{PCR}=$ polymerase chain reaction, $\mathrm{TREC}=\mathrm{T}$-cell receptor excision circle. 


\section{Box 3: Resources for physicians and families}

- Immune Deficiency Foundation (www.primaryimmune.org)

- Jeffrey Modell Foundation (www.info4pi.org)

- Severe Combined Immunodeficiency (www.scid.net)

- Immunodeficiency Canada (http://immunodeficiency.ca)

- Canadian Immunodeficiencies Patient Organization (www.cipo.ca)

- Rare Clinical Diseases Research Network: Primary Immune Deficiency Treatment Consortium (www.rarediseasesnetwork. org/cms/pidtc)

\section{Box 4: Unanswered questions}

- What is the best approach toward infants identified by newborn screening with idiopathic T cell lymphopenia who do not meet the criteria for severe combined immunodeficiency?

- Are there additional screening modalities that should be introduced into the newborn screen to identify other primary immunodeficiencies?

learning about an abnormal newborn screen, including grief, shock and fear of losing their child, and may not believe the results. ${ }^{17}$ Providers can ask directly about the emotional status of affected families and evaluate for postpartum depression. Social workers may provide psychosocial support to families and connect them with resources to facilitate additional medical visits, such as transportation assistance. Some useful resources for physicians and families are provided in Box 3.

\section{Conclusion}

The opportunity to identify severe combined immunodeficiency early in life has transformed outcomes for this otherwise fatal condition. ${ }^{10}$ Introducing this assay into newborn screening programs throughout Canada has the potential to save lives and prevent suffering of patients and families affected by this condition. Early treatment will decrease total clinical costs compared with those for children who are diagnosed and treated later in life. Continued evaluation of different cut-off levels for T-cell receptor excision circle and T-cell values will aid in arriving at best practices for use of the screen, and future research will aim to find new screening modalities capable of identifying additional primary immunodeficiencies that may also benefit from early recognition and treatment. Other unanswered questions are outlined in Box 4.

\section{References}

1. Turvey SE, Bonilla FA, Junker AK. Primary immunodeficiency diseases: a practical guide for clinicians. Postgrad Med J 2009;85:660-6.

2. Notarangelo LD. Primary immunodeficiencies. J Allergy Clin Immunol 2010;125: S182-94.

3. Adeli MM, Buckley RH. Why newborn screening for severe combined immunodeficiency is essential: a case report. Pediatrics 2010;126:e465-9.

4. Brown L, Xu-Bayford J, Allwood Z, et al. Neonatal diagnosis of severe combined immunodeficiency leads to significantly improved survival outcome: the case for newborn screening. Blood 2011;117:3243-6.
5. Pai SY, Logan BR, Griffith LM, et al. Transplantation outcomes for severe combined immunodeficiency, 2000-2009. N Engl J Med 2014;371:434-46.

6. Railey MD, Lokhnygina Y, Buckley RH. Long-term clinical outcome of patients with severe combined immunodeficiency who received related donor bone marrow transplants without pretransplant chemotherapy or post-transplant GVHD prophylaxis. J Pediatr 2009;155:834-40.e1.

7. Rozmus J, Junker A, Thibodeau ML, et al. Severe combined immunodeficiency (SCID) in Canadian children: a national surveillance study. J Clin Immunol 2013;33: 1310-6.

8. Buckley $\mathrm{RH}$. The long quest for neonatal screening for severe combined immunodeficiency. J Allergy Clin Immunol 2012;129:597-604.

9. Chan K, Puck JM. Development of population-based newborn screening for severe combined immunodeficiency. J Allergy Clin Immunol 2005;115:391-8.

10. Kwan A, Abraham RS, Currier R, et al. Newborn screening for severe combined immunodeficiency in 11 screening programs in the United States. JAMA 2014;312:729-38.

11. Stephan JL, Vlekova V, Ledeist F, et al. Severe combined immunodeficiency a retrospective single-center study of clinical presentation and outcome in 117 patients. J Pediatr 1993;123:564-72.

12. McWilliams LM, Railey MD, Buckley RH. Positive family history, infection, low absolute lymphocyte count (ALC), and absent thymic shadow: diagnostic clues for all molecular forms of severe combined Immunodeficiency (SCID). J Allergy Clin Immunol Pract 2015;3:585-91.

13. Rivers L, Gaspar HB. Severe combined immunodeficiency: recent developments and guidance on clinical management. Arch Dis Child 2015;100:667-72.

14. Villa A, Notarangelo LD, Roifman CM. Omenn syndrome: inflammation in leaky severe combined immunodeficiency. J Allergy Clin Immunol 2008;122:1082-6.

15. Shearer WT, Dunn E, Notarangelo LD, et al. Establishing diagnostic criteria for severe combined immunodeficiency disease (SCID), leaky SCID, and Omenn syndrome: the Primary Immune Deficiency Treatment Consortium experience. J Allergy Clin Immunol 2014;133:1092-8.

16. Verbsky J, Thakar M, Routes J. The Wisconsin approach to newborn screening for severe combined immunodeficiency. J Allergy Clin Immunol 2012;129:622-7.

17. Dorsey MJ, Dvorak CC, Cowan MJ, et al. Treatment of infants identified as having severe combined immunodeficiency by means of newborn screening. J Allergy Clin Immunol 2017;139:733-42.

18. Gaspar HB, Qasim W, Davies EG, et al. How I treat severe combined immunodeficiency. Blood 2013;122:3749-58.

19. Puck JM. Laboratory technology for population-based screening for severe combined immunodeficiency in neonates: the winner is T-cell receptor excision circles. J Allergy Clin Immunol 2012;129:607-16.

20. Verbsky JW, Baker MW, Grossman WJ, et al. Newborn screening for severe combined immunodeficiency; the Wisconsin experience (2008-2011). J Clin Immunol 2012;32:82-8.

21. Chien $\mathrm{YH}$, Chian SC, Chang KL, et al. Incidence of severe combined immunodeficiency through newborn screening in a Chinese population. J Formos Med Assoc 2015;114:12-6.

22. Somech R, Lev A, Simon AJ, et al. Newborn screening for severe T and B cell immunodeficiency in Israel: a pilot study. Isr Med Assoc J 2013;15:404-9.

23. de Felipe B, Olbrich P, Lucenas JM, et al. Prospective neonatal screening for severe T- and B-lymphocyte deficiencies in Seville. Pediatr Allergy Immunol 2016;27:70-7.

24. Barbaro M, Ohlsson A, Borte S, et al. Newborn screening for severe primary immunodeficiency diseases in Sweden - A 2-year pilot TREC and KREC screening study. $J$ Clin Immunol 2017;37:51-60.

25. Cross C. Ontario newborns now screened for SCID. CMAJ 2013;185:E616.

26. Ding Y, Thompson JD, Kobrynski L, et al. Cost-effectiveness/cost-benefit analysis of newborn screening for severe combined immune deficiency in Washington State. J Pediatr 2016;172:127-35.

27. Chan K, Davis J, Pai SY, et al. A Markov model to analyze cost-effectiveness of screening for severe combined immunodeficiency (SCID). Mol Genet Metab 2011;104:383-9.

28. Kwan A, Church JA, Cowan MJ, et al. Newborn screening for severe combined immunodeficiency and T-cell lymphopenia in California: results of the first 2 years. $J$ Allergy Clin Immunol 2013;132:140-50. 
29. van der Spek J, Groenwold RH, van der Burg M, et al. TREC-based newborn screening for severe combined immunodeficiency disease: a systematic review. J Clin Immunol 2015;35:416-30.

30. Shearer WT, Rosenblatt HM, Gelman RS, et al.; Pediatric AIDS Clinical Trials Group. Lymphocyte subsets in healthy children from birth through 18 years of age: the pediatric AIDS clinical trials group P1009 study. J Allergy Clin Immunol 2003;112: 973-80.

31. Accetta DJ, Brokopp CD, Baker MW, et al. Cause of death in neonates with inconclusive or abnormal T-cell receptor excision circle assays on newborn screening. J Clin Immunol 2011;31:962-7.

32. Baker M, Atkins A, Grossman W, et al. T-cell receptor excision circles of newborns are associated with gestational age: data from Wisconsin newborn screening for severe combined immunodeficiency. J Allergy Clin Immunol 2011; 127:AB146.

33. Kuo CY, Garcia-Lloret MI, Slev P, et al. Profound T-cell lymphopenia associated with prenatal exposure to purine antagonists detected by TREC newborn screening. J Allergy Clin Immunol Pract 2017;5:198-200.

34. Dasouki M, Okonkwo KC, Ray A, et al. Deficient T-cell receptor excision circles (TRECS) in autosomal recessive hyper IgE syndrome caused by DOCK8 mutation: implications for pathogenesis and potential detection by newborn screening. Clin Immunol 2011;141:128-32.
35. Mallott J, Kwan A, Church J, et al. Newborn screening for SCID identifies patients with ataxia telangiectasia. J Clin Immunol 2013;33:540-9.

36. Borte S, Fasth A, von Dobeln U, et al. Newborn screening for severe $T$ and B cell lymphopenia identifies a fraction of patients with Wiskott-Aldrich syndrome. Clin Immunol 2014;155:74-8.

37. Kwan A, Puck JM. History and current status of newborn screening for severe combined immunodeficiency. Semin Perinatol 2015;39:194-205.

38. Audrain M, Thomas C, Mirallie S, et al. Evaluation of the T-cell receptor excision circle assay performances for severe combined immunodeficiency neonatal screening on Guthrie cards in a French single-centre study. Clin Immunol 2014;150:137-9.

39. Lev A, Simon AJ, Broides A, et al. Thymic function in MHC class II-deficient patients. J Allergy Clin Immunol 2013;131:831-9.

40. Roifman CM, Somech R, Kavadas F, et al. Defining combined immunodeficiency. J Allergy Clin Immunol 2012;130:177-83.

41. Grazioli S, Bennett M, Hildebrand KJ, et al. Limitation of TREC-based newborn screening for ZAP70 severe combined immunodeficiency. Clin Immunol 2014;153:209-10.

42. Hiwarkar P, Gaspar HB, Gilmour K, et al. Impact of viral reactivations in the era of pre-emptive antiviral drug therapy following allogeneic haematopoietic SCT in paediatric recipients. Bone Marrow Transplant 2013;48:803-8.
Competing interests: Dr. Haddad reports grants from CSL Behring and consulting fees from Leadiant Biosciences, outside the submitted work. No other competing interests were declared.

This article has been peer reviewed.

Affiliations: Department of Pediatrics (Biggs, Turvey), British Columbia Children's Hospital, University of British Columbia, Vancouver, BC; Departments of Pediatrics, and Microbiology, Infection and Immunology (Haddad), University of Montreal, CHU Sainte-Justine, Montréal, Que.; Department of Pediatrics (Issekutz), IWK Health Centre, Dalhousie University, Halifax, NS; Division of Immunology and Allergy (Roifman), Hospital for Sick Children; Department of Pediatrics (Roifman), University of Toronto, Toronto, Ont.
Contributors: Catherine Biggs and Stuart Turvey contributed to the conception and design of the article, and drafted the manuscript. Elie Haddad, Thomas Issekutz and Chaim Roifman contributed to the design of the article and critically revised the article for important intellectual content. All of the authors gave final approval of the version to be published and agree to be accountable for all aspects of the work.

Funding: Catherine Biggs is supported by the Canadian Allergy, Asthma and Immunology Foundation/AllerGen Research Fellowship, the Primary Immune Deficiency Treatment Consortium grant for primary immune deficiencies and the AllerGen Emerging ClinicianScientist Research Fellowship. This work was supported by AllerGen NCE Inc. (the Allergy,
Genes and Environment Network), a member of the Networks of Centres of Excellence Canada program. The Primary Immune Deficiency Treatment Consortium (PIDTC; U54 AI 082973) is part of the National Center for Advancing Translational Science (NCATS) Rare Diseases Clinical Research Network, an initiative of the Office of Rare Diseases Research, NCATS. The PIDTC is funded through a collaboration between NCATS and the National Institute of Allergy and Infectious Diseases. Stuart Turvey holds the Aubrey J. Tingle Professorship in Pediatric Immunology and is a clinical scholar of the Michael Smith Foundation for Health Research.

Correspondence to: Stuart Turvey, sturvey@bcchr.ca 